\title{
Evidence for multiple scattering of high energy partons in nuclei
}

\section{E609 Collaboration}

\author{
M.D. Corcoran, R.K. Clark, K.A. Johns, M. Marcin, H.E. Miettinen, R.C. Moore, C. Naudet, \\ J.B. Roberts \\ T.W. Bonner Nuclear Laboratories, Rice University, Houston, TX 77251, USA
}

\author{
M.W. Arenton, W.R. Ditzler, T.H. Fields, G. Thomas \\ Argonne National Laboratory, Argonne, IL 60439, USA
}

\author{
M. Harrison \\ Fermilab, Batavia, IL 60510, USA
}

\author{
A. Kanofsky \\ Lehigh University, Bethlehem, PA 18015, USA \\ R. Gustafson \\ University of Michigan, Ann Arbor, MI 48109, USA
}

L. Cormell, M. Dris, J. Fleischman, W. Kononenko, B. Robinson, G Theodosiou, W. Selove, B. Yost

University of Pennsylvania, Philadelphia, PA 19104, USA

H.F. Chen, A.R. Erwin, M.A. Hasan, C.E. Kuehn, K.S. Nelson and M.A. Thompson

University of Wisconsin, Madison, WI 53706, USA

Received 24 November 1990

\begin{abstract}
New experimental information is presented about the effect of a nucleus on partons which have undergone a hard scatter. We have observed clear dijet events from $400 \mathrm{GeV} / \mathrm{c}$ p-nucleus interactions and compared them to dijets from pp interactions. Our results show that the angular width of the individual jets is only slightly affected by the presence of the nucleus, but the acoplanarity of the jet pairs is sharply increased.
\end{abstract}

\section{Introduction}

The "anomalous nuclear enhancement" effect dates from the experiments of Cronin et al. in 1975 [1]. In those experiments it was observed that the cross section for producing high- $p_{\mathrm{t}}$ single particles increased with nuclear mass as $A^{\alpha}$, with $\alpha$ ranging from $1.1-$ 1.4 and varying with particle type. Numerous explanations have been suggested for this effect, but the most widely accepted is secondary scattering of partons inside the nucleus after a hard scatter [2]. However, there has been no direct experimental measurement of this secondary scattering.

In this letter we present new experimental information about the effect of a nucleus on partons which have undergone a hard scatter. We clearly observe dijet events from heavy nuclei and compare them quantitatively to dijets from hydrogen. 


\section{Apparatus and analysis}

In Fermilab experiment $\mathrm{E} 609,400 \mathrm{GeV} / c$ protons were incident on a hydrogen target or one of seven nuclear targets ( $\mathrm{He}, \mathrm{Be}, \mathrm{C}, \mathrm{Al}, \mathrm{Cu}, \mathrm{Sn}$, and $\mathrm{Pb}$ ). The solid targets were mounted on a wheel which changed positions every beam spill. The E609 detector has been described in detail elsewhere [3]. The main calorimeter consists of 132 towers of lead-scintillator and iron-scintillator sampling calorimeter. Its center-ofmass (CM) polar angular range is $30^{\circ}-130^{\circ}$, and the entire azimuth is covered. An array of wire chambers upstream of the calorimeter was used for charged particle tracking. All events in this analysis have been tracked and required to yield a production vertex in the target.

It is well known that at fixed-target energies a large solid-angle transverse energy trigger (the "global" $E_{\mathrm{t}}$ trigger) is not a selective dijet trigger [4]. We and other groups have used "limited solid angle" triggers to trigger on and measure both single jet and dijet events at fixed target and ISR energies. Such a trigger requires $E_{\mathrm{t}}$ above a set threshold in some subregion of the full calorimeter, usually on the order of one steradian solid angle per jet. Our Monte Carlo studies [5] indicate that such triggers are efficient jet triggers with good background rejection. However, such a trigger can introduce a geometrical bias by requiring $E_{1}$ in a specific solid angle. For dijet events the biases can be more complex, since the two jets in general need not be back to back in either azimuthal or polar angle.

We have devised a general type of dijet trigger which we call the "two-high" trigger. This trigger requires the $E_{1}$ of any two towers in the calorimeter to be above a threshold of about $1.0 \mathrm{GeV}$. This trigger has little geometric bias, since no requirement is placed on the relative location of the triggering towers. It is insensitive to low- $p_{\mathrm{t}}$ fragments from the spectator jets or soft gluon radiation, but is sensitive to jet events, since they tend to deposit a large amount of $E_{\mathrm{t}}$ in a small solid angle. Our Monte Carlo studies and other studies using experimental data $[3,5,6]$ indicate that, using this trigger and then requiring a standard jet-finding algorithm to find at least two jets of average $p_{\mathrm{t}}>4 \mathrm{GeV} / c$, the non-jet background is negligible. We have previously published pp jet cross sections using the two-high trigger [6] as well as solid angle triggers [7]. All of the data used in this analysis was obtained with the two-high trigger with a hardware cut of about $1 \mathrm{GeV}$ in $E_{\mathrm{t}}$ on the triggering towers. Software $E_{\mathrm{t}}$ cuts of 1.9 and $1.6 \mathrm{GeV}$ on the hottest and second hottest towers were applied off-line.

The geometric pattern of energy deposition in the calorimeter was deconvoluted offline to make calorimeter "clusters", which are an approximation to particle momenta. This procedure is not perfect, and the clustering algorithm will inevitably combine the showers of hadrons which are close to each other in space into one cluster. Also, softer hadrons tend to be clustered together with a more energetic hadron. Nevertheless, our Monte Carlo studies have shown that the "found" jets are closer to the "true" jets both in $p_{\mathrm{t}}$ and angle when the jet-finding algorithms operate on the clusters rather than the raw calorimeter towers. The results presented here have been obtained treating calorimeter clusters as massless particles, with the jet-finders operating on the particles.

Two very different jet-finding algorithms have been used. The first [6] used a cone of fixed half angle and maximized the vector $p_{1}$ of the particles within that cone. The half-angle of the cone has been chosen from Monte Carlo studies to be $45^{\circ}$. The second jet-finding algorithm is based on a method developed at the ISR [8]. This algorithm operates on a $p_{t}$ surface in rapidity-azimuth space which is calculated as a sum of gaussian contributions over all particles in the event. The height of the surface is taken to be the $p_{1}$ of the jet.

We used these two different algorithms to study the sensitivity of our results to the jet-finders, and found that for all the results presented here there is no significant difference in results from the two methods.

Events in which at least two jets were found with $\mathrm{CM}$ angles in the range $60^{\circ}-110^{\circ}$ with an average $p_{\mathrm{t}}>4 \mathrm{GeV} / c$, were kept for further analysis. For $\mathrm{H}$ $(\mathrm{Pb})$ there were a total of $1400(366)$ events surviving these cuts. The other nuclear targets all had fewer events than $\mathrm{Pb}$.

\section{Jets from pp interactions}

The azimuthal distribution of $E_{\mathrm{t}}$ flow for dijet events with average $p_{\mathrm{t}}>4 \mathrm{GeV} / c$ is shown by the solid histogram in fig. 1a. $\phi=0$ is defined here by the azi- 
muthal angle of the higher momentum jet. The second jet appears to be much more diffuse, but in fact that is an effect of acoplanarity rather than jet size. This point is illustrated by the dotted histogram in fig. 1a which shows the $E_{1}$ flow when the second jet defines $\phi=0$. The second jet is clearly seen to have practically the same width as the more energetic jet. The opposite jet appears more diffuse for both curves because the two jets are not back-to-back in the transverse plane, so that their azimuthal angle difference $(\Delta \phi)$ varies from event to event.

The $E_{1}$ flow near $\phi=0$ in fig. 1a is biased toward
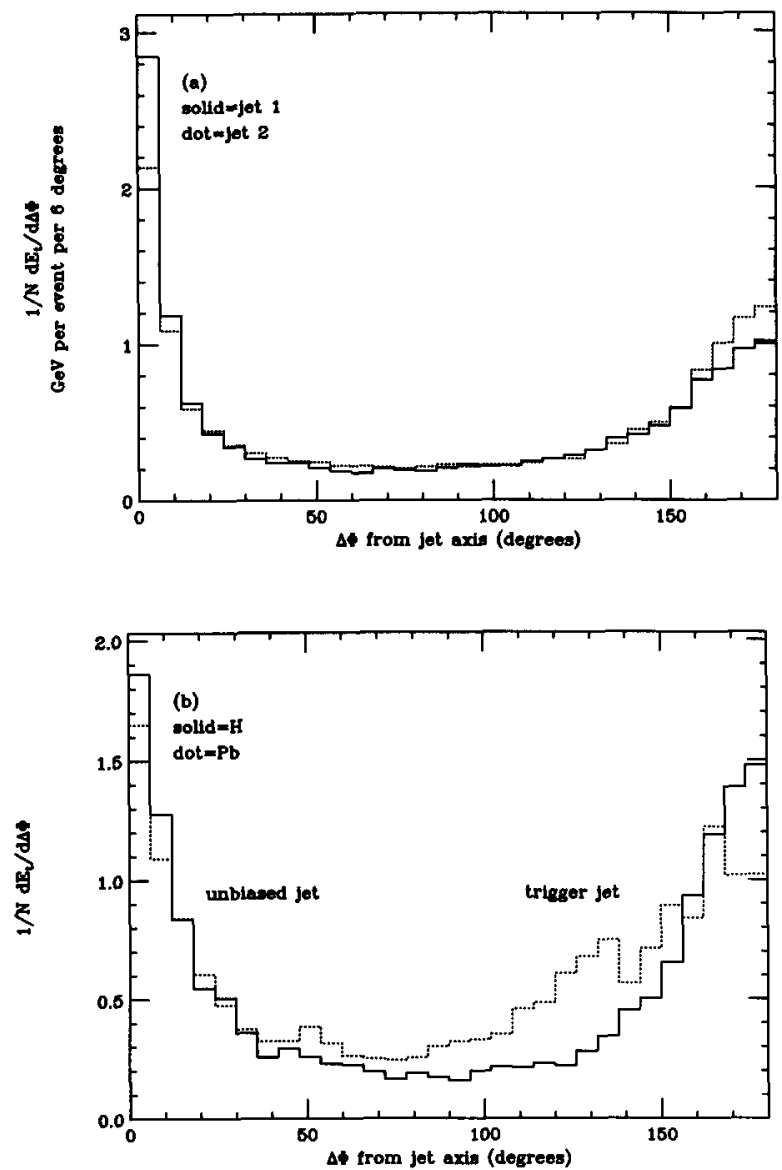

Fig. 1. Azimuthal distribution of transverse energy for dijet events with $p_{\mathrm{t}}>4 \mathrm{GeV} / c$. (a) Dijets from $\mathrm{H}$. Solid: $\phi=0$ is defined by the direction of the higher $p_{\mathrm{t}}$ jet. Dotted: $\phi=0$ is defined by the lower $p_{\mathrm{t}}$ jet. (b) Solid: dijets from $\mathrm{H}$, with $\phi=0$ defined by the direction of the unbiased jet (see text). Dotted histogram: same as solid, except using $\mathrm{p}-\mathrm{Pb}$ events. narrower jets by the two-high (tower) trigger requirement described above. To measure the true jet angular width without this trigger bias effect, we have selected events in which the trigger condition was satisfied completely within one jet. Then the angular width of the other jet will not be directly affected by the trigger requirement. The solid curve in fig. $1 \mathrm{~b}$ shows the $E_{1}$ flow for this subset of all events, with $\phi=0$ now defined by the found direction of the "unbiased" jet. The jet structure is still very apparent, and the RMS jet width is now about $14^{\circ}$. This can be compared to a value of about $8^{\circ}$ from the AFS jet analysis [9] which was at a higher $p_{\mathrm{t}}(9 \mathrm{GeV} / c$ rather than $4 \mathrm{GeV} / \mathrm{c}$ ).

The $E_{\mathrm{t}}$ flow shown in figs. $1 \mathrm{a}$ and $1 \mathrm{~b}$ was calculated using calorimeter clusters as particles. We have also plotted the same distributions using only the raw tower $E_{\mathrm{t}}$ 's for both jet-finding and $E_{\mathrm{t}}$ flow. We find that the apparent width of the jets increases by about $3^{\circ}$ as would be expected due to the spreading of hadron showers. However the jet structure is still clear.

The solid curve in fig. 2a shows the distribution in azimuthal angle between the two jets $(\Delta \phi)$ for pp events. The width of this peak is a measure of $k_{t}$, the vector unbalance in $p_{\mathrm{t}}$ of the two jets. Several physical effects contribute to $k_{\mathfrak{t}}$, including initial and final state gluon radiation as well as rescattering in a nucleus. By doing a gaussian fit to the $\Delta \phi$ distribution near $\Delta \phi=180^{\circ}$, we can extract the standard deviation of this distribution. We define $k_{11}$ as the component of $k_{\mathrm{t}}$ which is in the $\phi$ direction. We estimate $k_{\mathrm{t} 1}$ as $p_{\mathrm{t}} \sin \sigma$, where $\sigma$ is the standard deviation of the $\Delta \phi$ distribution and $p_{\mathrm{t}}$ is the average $p_{\mathrm{t}}$ of the two found jets. We find that $k_{\mathrm{t}}$ has an RMS value of $0.9 \pm 0.2$ $\mathrm{GeV} / c$. We have also calculated the RMS value of $k_{t 1}$ by calculating $p_{\mathrm{t}} \sin \Delta \phi$ event-by-event and find good agreement between the two methods. We have applied a correction for the expected uncertainty in the found jet direction due to errors in jet reconstruction. Our result for $k_{\mathrm{t}}$ is comparable to that from the AFS dijet experiment, which quotes a value of 1.0 $\mathrm{GeV} / c[9]$.

In calculating $k_{\mathrm{t} 1}$ by either method, we have excluded events with $\Delta \phi$ less than a cutoff, chosen to be the point at which the $\Delta \phi$ distribution has fallen to $20 \%$ of its maximum value. The reason for the cutoff was to exclude events with very large values of $k_{\mathrm{t}}$; there are few of these events, but they can have a large 

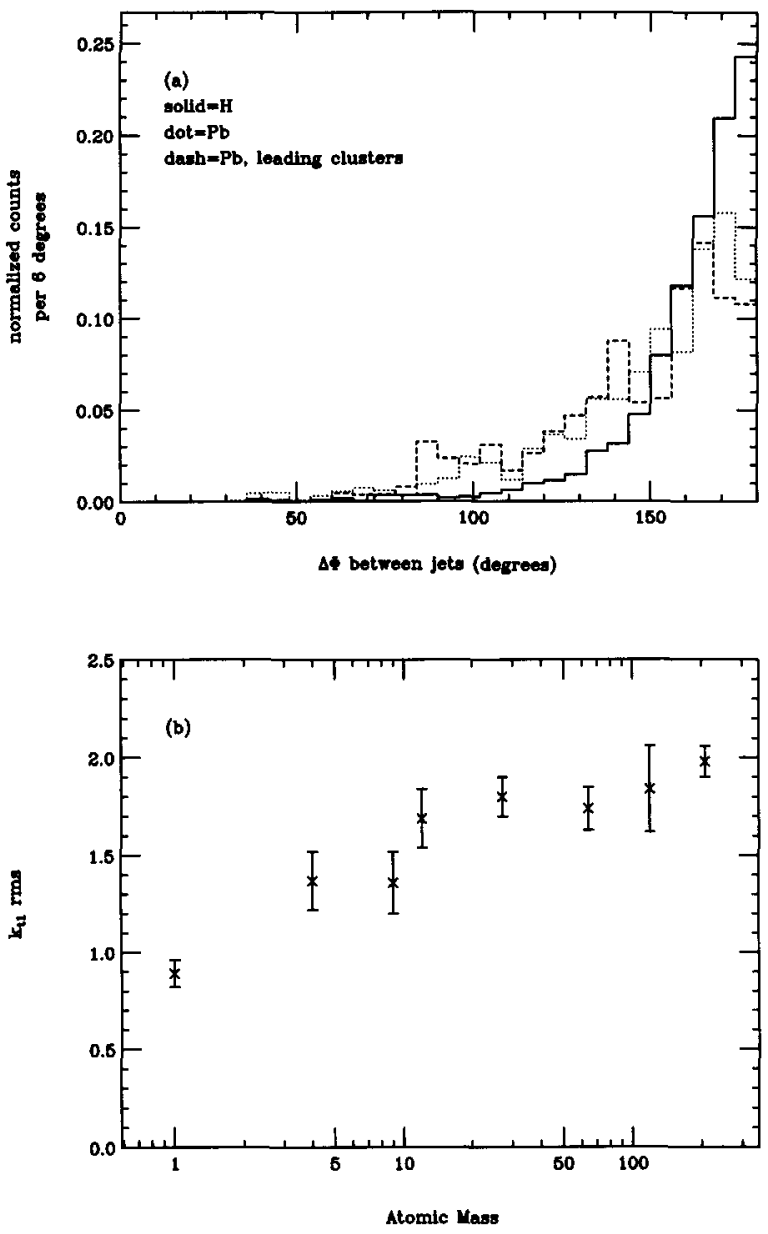

Fig. 2. (a) Distribution of $\Delta \phi$, the azimuthal angle between the jet axes. The energy flow is plotted using calorimeter clusters. Solid: $\mathrm{H}$ events; dotted: $\mathrm{Pb}$ events; dashed: $\mathrm{Pb}$ events, using the azimuthal angles of the leading clusters. (b) RMS value of $k_{\mathbf{t 1}}$ (defined in the text) as a function of atomic mass $A$. Errors shown are statistical only. Each point has a systematic error of $0.2 \mathrm{GeV} /$ $c$.

effect on the mean value. This cutoff angle was $145^{\circ}$ for hydrogen. We estimate a systematic error in $k_{\mathrm{tl}}$ of $0.2 \mathrm{GeV} / c$ due to the somewhat arbitrary choice of the cutoff.

We are assuming in the calculation of $k_{\mathrm{t}}$ that the magnitudes of the two jet $p_{t}$ 's are roughly the same, which in fact is true. For pp interactions, the magnitudes of the jet $p_{\mathrm{t}}$ 's differ by about $20 \%$ on the average.

\section{Jets from heavy nuclei}

The same analysis procedures have been applied to data from heavy nuclei. The dotted histogram in fig. lb shows the $E_{\mathrm{t}}$ flow for p-Pb events for unbiased jets as defined above. Three features stand out. First, the jets from $\mathrm{Pb}$ are very similar to those from $\mathrm{H}$, their RMS width being slightly larger $\left(17^{\circ}\right.$ versus $14^{\circ}$ for $\mathrm{H})$. Second, it can be seen that the transverse energy density near $\phi=90^{\circ}$ is higher by about $40 \%$ for $\mathrm{Pb}$ (We have corrected for this effect in calculating jet $p_{\mathrm{t}}$ 's for $\mathrm{Pb}$ ). The third and most pronounced effect is the increased acoplanarity for $\mathrm{Pb}$ compared to $\mathrm{H}$. The dotted histogram in fig. 2a shows the distribution of $\Delta \phi$ between the jets for $\mathrm{Pb}$. The broadening compared to $\mathrm{H}$ is dramatic, with the standard deviation of the $180^{\circ}$ peak increasing from $13^{\circ}$ for $\mathrm{H}$ to $24^{\circ}$ for $\mathrm{Pb}$. The RMS value of $k_{\mathrm{t} 1}$ (calculated by two methods, as described above) for the Pb events is $2.0 \pm 0.2$ $\mathrm{GeV} / c$. In calculating $k_{\mathrm{t}}$, the cutoff in $\Delta \phi$ for $\mathrm{p}-\mathrm{Pb}$ events (as described above) was $115^{\circ}$. The difference in the magnitudes of the jet $p_{\mathrm{t}}$ 's for $\mathrm{Pb}$ events was $25 \%$, slightly larger than that for hydrogen. Fig. $2 \mathrm{~b}$ shows $k_{11}$ RMS versus $A ; k_{11}$ appears to increase smoothly with $A$.

An important difference between hydrogen and nuclear target events is the total charged multiplicity as measured by the wire chambers. For $\mathrm{H}$ jet events the average is 15 , while for $\mathrm{Pb}$ the average is 24 . However, the extra particles are rather soft, since the total main calorimeter (lab) energy increases by only about $35 \mathrm{GeV}$ from $\mathrm{H}(248 \mathrm{GeV})$ to $\mathrm{Pb}(283 \mathrm{GeV})$. The extra energy in the main calorimeter was found to be primarily at $\mathrm{CM}$ angles larger than $60^{\circ}[10,11]$.

One question immediately comes to mind. Could the observed differences in jet acoplanarity be simply an artifact of these extra soft particles? They increase the transverse energy of the underlying event and will therefore affect the ability of the jet-finders to correctly measure the jet $p_{1}$ and angles.

One method we have used to answer this question is to look at the direction, not of the total jet, but of the "leading clusters" (the cluster with the largest fractional momentum or $z$ ). The direction of the leading cluster is insensitive to the presence of additional soft particles in the event. The dashed histogram in fig. 2a shows $\Delta \phi$ for the leading clusters for lead events. The distributions for the full jet and the 
leading clusters are seen to be very similar. We have also checked that the directions of the triggering towers exhibit the same behavior.

\section{Fluctuation Monte Carlo}

A second independent method to evaluate the influence of the additional soft particles was a "fluctuation Monte Carlo". We have taken actual $\mathrm{H}$ dijet events and added $35 \mathrm{GeV}$ additional energy in the main calorimeter in order to simulate $\mathrm{Pb}$ events. We have used the observed energy flows and charged multiplicities to determine on the average the number of extra particles and where in polar angle their extra energy should be deposited in the calorimeter. However, fluctuations about these averages can occur on an event-by-event basis, possibly resulting in large changes in the "found" jets. This is the effect we are trying to estimate. After the extra energy was deposited into the calorimeter, we reapplied the jetfinders. Events were selected in which the two-high trigger requirement was satisfied within one jet, as in fig. 1 b. Fig. 3 compares the azimuthal $E_{\mathrm{t}}$ flow for $\mathrm{H}$ data with this "fluctuation Monte Carlo". It is clear by comparison with fig. $1 \mathrm{~b}$ that the extra energy and multiplicity in the underlying event do not by themselves account for the large acoplanarity seen in the

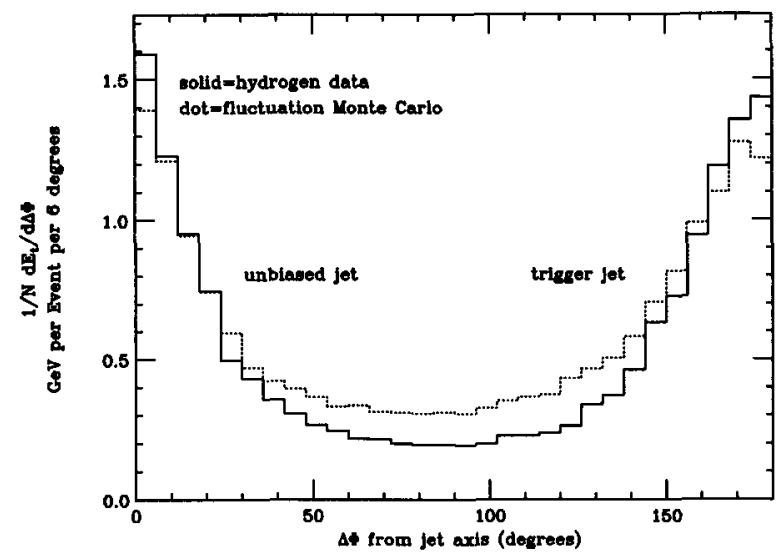

Fig. 3. Azimuthal distribution of transverse energy for unbiased jet selection, as discussed in the text. The energy flow is plotted using calorimeter towers rather than clusters. Solid: $H$ data (compare to fig. 1b). dotted: result of "fluctuation Monte Carlo" calculation (see text).
$\mathrm{Pb}$ data. The calculated value of $k_{\mathrm{tl}}$ increased from $0.9 \mathrm{GeV} / c$ to $1.05 \mathrm{GeV} / c$ for these "fluctuation" Monte Carlo events, much smaller than the observed difference between $\mathrm{H}$ and $\mathrm{Pb}$. However, the $E_{\mathrm{t}}$ increase in the region between the jets is well accounted for by the addition of the extra $35 \mathrm{GeV}$ of energy.

\section{Planarity and A-dependence of cross sections}

A variable which has often been used to quantify the "jettiness" of events is planarity. Planarity is calculated using only momentum components in the plane perpendicular to the beam direction. An axis is found which maximises the sum of the squares of all momentum components ( $b_{\max }$ ) along that axis while minimizing the sum of the squares of momentum components perpendicular to that axis $\left(b_{\min }\right)$. Planarity is then defined as $P=\left(b_{\max }-b_{\min }\right) /\left(b_{\max }+\right.$ $\left.b_{\text {min }}\right)$. For two narrow back-to-back jets $P$ approaches 1 , while for a circularly symmetric event $P$ is 0 .

Fig. 4a shows the planarity distributions for the same $\mathrm{H}$ and $\mathrm{Pb}$ dijet events shown in previous plots. Note the significant downward shift of the planarity distribution for $\mathrm{Pb}$. This shift occurs despite the fact that the individual jets from lead have nearly the same angular width as those from hydrogen, as shown by fig. $1 \mathrm{~b}$. Fig. $4 \mathrm{~b}$ shows the planarity distribution from the fluctuation Monte Carlo events described above. The additional soft particles do shift the planarity distribution downward, but not by nearly as much as the observed difference between $\mathrm{H}$ and $\mathrm{Pb}$. We conclude that the dominant cause of the lowered planarity is the increased $\Delta \phi$ of the jet pairs and not a broadening of the individual jets or the presence of additional soft particles. From these results it is clear that a cut on planarity to isolate jet-like events from nuclei is likely to be an extremely biased cut. The observed increased acoplanarity of the jets from $\mathrm{Pb}$ is reflected in the observed decreased planarity. A planarity requirement will reject an $A$-dependent fraction of otherwise good dijet events.

The cross section behavior will be discussed in detail in a separate paper. We will just summarize the results by noting that we do observe an anomalous nuclear enhancement, with $\alpha$ equal to about $1.3 \pm 0.1$ for jets of $p_{1}>4 \mathrm{GeV} / c$. However, a word of caution is in order. Any kinematic cuts used to isolate a 

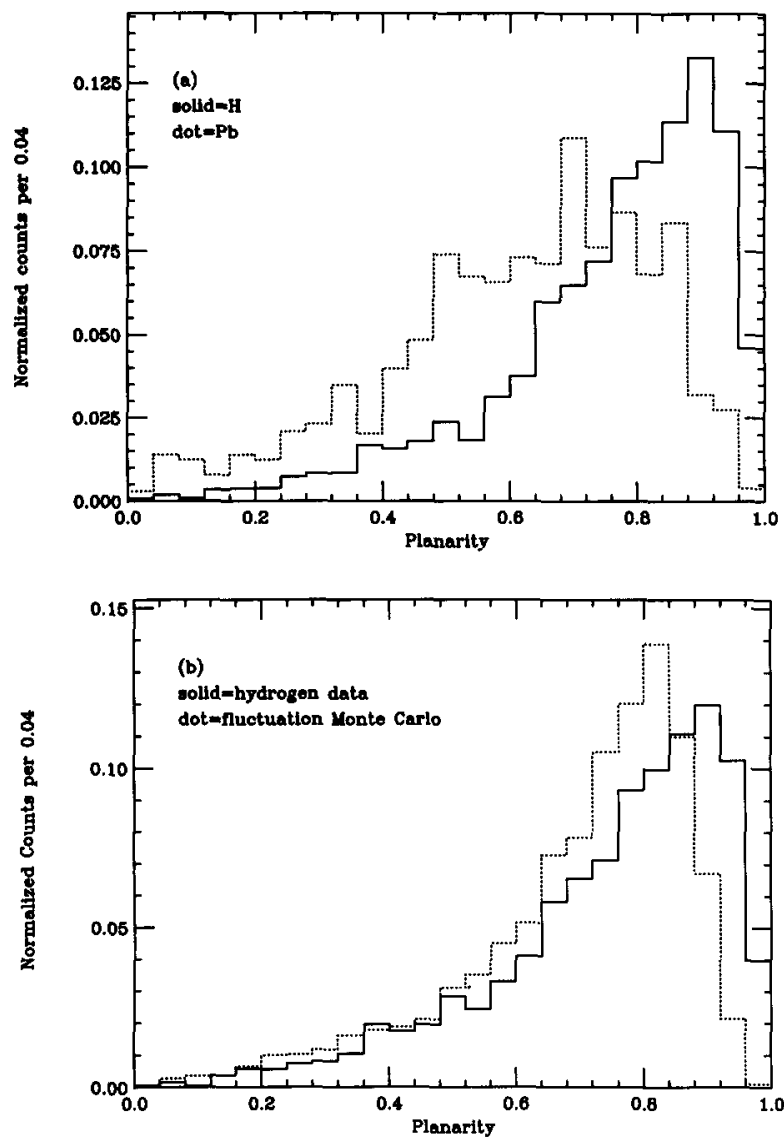

Fig. 4. Planarity distributions for dijet events with $p_{\mathfrak{\imath}}>4 \mathrm{GeV} / c$. (a) Solid $=\mathrm{H}$ data, dotted $=\mathrm{Pb}$ data. (b) Comparison of $\mathrm{H}$ data and "fluctuation Monte Carlo" events.

"cleaner" jet sample can influence the value of $\alpha$. For example, $\alpha$ will decrease sharply if one requires planarity $>0.7$ (say). In general, one would expect that any kinematic cuts which require events to be more hydrogen-like will reduce $\alpha$, since such cuts bias against nuclear events in which the partons have undergone significant secondary scattering. Our data are consistent with this expectation.

\section{Other data}

Another experiment with similar data is E557 at Fermilab [12]. That experiment was done at a higher beam momentum $(800 \mathrm{GeV} / \mathrm{c})$ and therefore has a larger $p_{1}$ range than E609. The E557 group used a limited solid angle trigger rather than a two-high trigger. Their trigger geometry required the sum of two triggering sections, $180^{\circ}$ apart in azimuth and each covering $90^{\circ}$ of azimuth and polar angles of $45^{\circ}-135^{\circ}$ to be above a threshold.

E557 reports a broadening of the distribution of azimuthal angle between the jets for heavy nuclei. However, because their triggering regions are centered $180^{\circ}$ apart in azimuth, and perhaps because of their higher jet momentum, it is not surprising that the E557 results do not show as dramatic an increase in acoplanarity as we observe. This points to an important advantage of the two-high trigger: it is geometrically unbiased and will trigger on the jets wherever they are in the calorimeter.

The E557 group reports an $\alpha$ of 1.5 for dijet production, dropping slowly to 1.25 with increasing $p_{\mathrm{t}}$. After a correction for the underlying event, they report a value of $\alpha$ of about 1.1. This result is not in contradiction to ours, since their trigger is insensitive to events with large values of $\Delta \phi$. E557 observes a sensitivity of $\alpha$ to kinematic cuts and a slight widening of their observed jets, similar to our results.

\section{Conclusion}

We have clearly observed dijet events from nuclei ranging from $\mathrm{H}$ to $\mathrm{Pb}$. We find that the angular size of individual jets from heavy nuclei is little different than from hydrogen. However, we do observe a dramatic change in the coplanarity of the two jets in dijet events. For $\mathrm{H}$, we find an RMS value for $k_{11}$ of $0.9 \pm 0.2 \mathrm{GeV} / c$, which increases to $2.0 \pm 0.2 \mathrm{GeV} / c$ for $\mathrm{Pb}$. This large increase in $k_{11}$ is directly reflected in a substantial downward shift in the dijet planarity distribution for a heavy nucleus. Random fluctuations in the underlying p-nucleus event do not account for this large increase in $k_{\mathbf{t}}$.

Our results appear to be in qualitative accord with expectations from a parton multiple scattering mechanism, but our value of $k_{11}$ for $\mathrm{Pb}$ may be difficult to explain quantitatively. For example, our value of $k_{\mathrm{t}}$ is much larger than measured (for the incident parton) in Drell-Yan dilepton production [13]. However, these results may be not contradictory, since one might expect the interactions of the outgoing final 
state partons (which are separating from each other as they leave the nucleus) to be quite different from the interactions of incoming partons which are bound in a hadron.

\section{Acknowledgement}

This work was supported in part by the US Department of Energy.

\section{References}

[1] L. Kluberg et al., Phys. Rev. Lett. 38 (1977) 670; D. Antreasyan et al., Phys. Rev. D 19 (1979) 764.
[2] See e.g., G. Farrar, Phys. Lett. B 56 (1975) 185; M. Lev and B. Petersson, Z. Phys. C 21 (1983) 155.

[3] M.W. Arenton et al., Phys. Rev. D 31 (1985) 984; K.A. Johns, M.A. thesis, Rice University (1983), unpublished.

[4] C. de Marzo et al., Phys. Lett. B 112 (1982) 173.

[5] T. Fields, R. Singer and W. Selove, Phys. Rev. D 25 (1982) 2451; M.D. Corcoran, Phys. Rev. D 32 (1985) 592.

[6] M.W. Arenton et al., Phys. Rev. Lett. 53 (1984) 1988.

[7] L.R. Cormell et al., Phys. Lett. B 150 (1985) 322; K.S. Nelson et al., Nucl. Phys. B 294 (1987) 1022.

[8] M.G. Albrow et al., Nucl. Phys. B 160 (1979) 1.

[9] T. Akesson et al., Z. Phys. C 30 (1986) 27.

[10] R.C. Moore, Ph. D. thesis, Rice University (1989), unpublished.

[11 ] H. Miettinen et al., Phys. Lett. B 207 (1988) 222; R.C. Moore et al., Phys. Lett. B 244 (1990) 347

[12] C. Stewart et al., Phys. Rev. D 42 (1990) 1385.

[13] See e.g., P. Bordalo et al., Phys. Lett. B 193 (1987) 373. 